\title{
Editorial
}

\section{The International Journal of Astrobiology}

The launch of a new journal is appropriately like a space mission. It is the result of a scientific need, the inspiration of a group of committed scientists and technologists, a series of draft proposals, an approved mission protocol, and a launch. Today is the launch day for a journal whose remit has only recently consolidated from diverse disciplines. Cambridge University Press has an international reputation for astronomy. To this we add extreme biology and its associated environmental research to integrate astrobiology as: 'the study of the origin, evolution, adaptation and distribution of past and present life in the Universe'.

Astrobiology has three main themes: (1) Origin, evolution and limits of life on Earth; (2) Future of life, both on Earth and elsewhere; (3) Search for habitats, biomolecules and life in the Solar System and elsewhere. These fundamental concepts require the integration of various disciplines, including biology (especially microbiology), chemistry, geology, palaeontology, and the physics of atmospheres, planets and stars. We must also keep our minds wide open about the nature and limits of life. We can safely assume a carbon-based system within Solar Systems as we know them, but our concept of habitable zones expands yearly. We were taught that only the spores of certain bacilli could survive temperatures above the boiling point of water, and yet we now know that the deep-sea vent microbe Pyrolobus can survive an hour at $121{ }^{\circ} \mathrm{C}$, which is the temperature used for sterilising medical instruments. We know of cyanobacteria which can not only live inside deep-frozen Antarctic rocks but also survive on roof-tops in Jerusalem at $80{ }^{\circ} \mathrm{C}$. The bacterium Deinococcus radiodurans tolerates lethal doses of nuclear radiation, and cyanobacteria inside Antarctic desert sandstone receive so little moisture that their carbon turnover time (from its fixation by photosynthesis to its release as carbon dioxide during respiration) is 10,000 years. Life is tolerant, adaptable and tenacious.

The 'habitable zone' is defined by biological parameters, but we also need to define the physical and chemical conditions for life in stellar and planetary environments. This is where biologists, physicists, chemists and geologists (in the broadest sense) meet synergistically. This essential collaboration encompasses topics ranging from the interaction of minerals and organic building blocks for synthesising biomolecules to the search for extra-solar planets. These may have distant habitable zones which could have progressed much further down the evolutionary road than our own planet, Earth. There is also a time dimension in all this. We must not only consider present forms of life, but also its origins and palaeological record if we are to predict its likely occurrence and evolution elsewhere.

Astrobiology is alive and well. This new International Journal of Astrobiology will foster the interaction between scientists world-wide who now recognise that their 
diverse interests can contribute to the discipline. Its launch is the beginning of a new mission for the benefit of all on this planet (and others?).

David D. Wynn-Williams Project Leader, Antarctic Astrobiology British Antarctic Survey 12 February 2002 